\title{
ON THE CONVERGENCE BETWEEN THE MANN ITERATION AND ISHIKAWA ITERATION FOR THE GENERALIZED LIPSCHITZIAN AND \$-STRONGLY PSEUDOCONTRACTIVE MAPPINGS
}

\author{
ZHiQun Xue
}

\begin{abstract}
In this paper, we prove that the equivalence between the convergence of Mann and Ishikawa iterations for the generalized Lipschitzian and $\Phi$-strongly pseudocontractive mappings in real uniformly smooth Banach spaces. Our results significantly generalize the recent known results of [B. E. Rhoades and S. M. Soltuz, The equivalence of Mann iteration and Ishikawa iteration for non-Lipschitz operators, Int. J. Math. Math. Sci. 42 (2003), 2645-2651].
\end{abstract}

\section{Introduction}

Let $E$ be a real Banach space. Let $J$ denote the normalized duality mapping from $E$ to $2^{E^{*}}$ defined by

$$
J x=\left\{f \in E^{*}:\langle x, f\rangle=\|x\| \cdot\|f\|=\|f\|^{2}\right\},
$$

where $E^{*}$ denotes the dual space of $E$ and $\langle\cdot, \cdot\rangle$ denotes the generalized duality pairing. It is well known that if $E$ is a uniformly smooth Banach space, then $J$ is single-valued, $J(t x)=t J(x)$ for all $x \in E$ and $t \geq 0$, and $J$ is uniformly continuous on any bounded subset of $E[1,3]$. We denote the single-valued normalized duality mapping by $j$.

A map $T$ with domain $D(T)$ and range $R(T)$ in $E$ is said to be strongly pseudocontractive if there exists a constant $k \in(0,1)$ such that for any $x, y \in$ $D(T)$, there exists $j(x-y) \in J(x-y)$ such that

$$
\langle T x-T y, j(x-y)\rangle \leq k\|x-y\|^{2} .
$$

The map $T$ is called $\Phi$-strongly pseudocontractive if, for all $x, y \in D(T)$, there exist $j(x-y) \in J(x-y)$ and a strictly continuous increasing function

Received June 4, 2007; Revised August 29, 2008.

2000 Mathematics Subject Classification. 47H09, 47H10, 47H17.

Key words and phrases. Ishikawa iteration, Mann iteration, $\Phi$-strongly pseudocontractive maps, generalized Lipschitz maps, uniformly smooth Banach spaces.

The Project is supported by the National Natural Science Foundation of China Grant 10872136 and Hebei province science and technology foundation(No. 072056197D). 
$\Phi:[0,+\infty) \rightarrow[0,+\infty)$ with $\Phi(0)=0$ such that

$$
\langle T x-T y, j(x-y)\rangle \leq\|x-y\|^{2}-\Phi(\|x-y\|)\|x-y\| .
$$

Set $\Phi(t)=k t, t \in[0,+\infty)$ and $k$ is a constant of $(0,1)$, we get the above definition of strongly pseudocontractive mapping.

The mapping $T: D(T) \subset E \rightarrow E$ is called generalized Lipschitz map if there exists a constant $L>0$ such that

$$
\|T x-T y\| \leq L(1+\|x-y\|)
$$

for all $x, y \in D(T)$. Clear, if map $T$ either is Lipschitz or has a bounded range, then $T$ must be a generalized Lipschitz map. Conversely, the following example indicates that the class of generalized Lipschitz map neither is Lipschitz nor has the bounded range.

Example. Let $E=(-\infty,+\infty)$ with the usual norm. Define $T: E \rightarrow E$ by

$$
T x=\left\{\begin{array}{cl}
\frac{-3 x}{2}+1, & \text { if } x \in(-\infty,-1), \\
1, & \text { if } x=-1, \\
\frac{-3 x}{2}+\sqrt{-x}, & \text { if } x \in(-1,0), \\
\frac{-3 x}{2}, & \text { if } x \in[0,+\infty) .
\end{array}\right.
$$

Then $T$ is not continuous when $x=-1$; However $T$ is both a generalized Lipschitz map with $0 \in \operatorname{Fix}(T)$ and a strongly pseudocontractive map. Of course, $T$ is also a $\Phi$-strongly pseudocontractive map.

Let $D$ be a nonempty closed convex subset of $E$ and $T: D \rightarrow D$. Suppose that $u_{0}, x_{0} \in D$ are arbitrary. The Mann iteration is defined by

$$
u_{n+1}=\left(1-a_{n}\right) u_{n}+a_{n} T u_{n}, n \geq 0,
$$

where $\left\{a_{n}\right\}_{n=0}^{\infty} \in[0,1]$. The Ishikawa iteration is defined by

$$
\left\{\begin{array}{c}
y_{n}=\left(1-b_{n}\right) x_{n}+b_{n} T x_{n}, n \geq 0 \\
x_{n+1}=\left(1-a_{n}\right) x_{n}+a_{n} T y_{n}, n \geq 0
\end{array}\right.
$$

where $\left\{a_{n}\right\}_{n=0}^{\infty}$ and $\left\{b_{n}\right\}_{n=0}^{\infty}$ are two real sequences of $[0,1]$.

Recently, B. E. Rhoades and S. M. Soltuz [6] established the following results of equivalence of iterations convergence.

Theorem R-S1 ([6, Theorem 2.1]). Let $X$ be a real Banach space with a uniformly convex dual and $B$ a nonempty, closed, convex, and bounded subset of $X$. Let $T: B \rightarrow B$ be a continuous and strongly pseudocontractive operator. Then for $u_{1}=x_{1} \in B$, the following assertions are equivalent:

(i) Mann iteration (1.1) converges to the fixed point of T;

(ii) Ishikawa iteration (1.3) converges to the fixed point of $T$.

Theorem R-S2 ([6, Corollary 3.1]). Let $X$ be a real Banach space with a uniformly convex dual and $B$ a nonempty, convex and closed subset of $X$. Let $S: B \rightarrow B$ be a continuous and strongly accretive operator and let $\left\{x_{n}\right\}_{n}$, 
given by (3.2), be bounded. Then, for $u_{1}=x_{1} \in B$, the following assertions are equivalent:

(i) Mann iteration (3.3) converges to the solution of $S x=f$;

(ii) Ishikawa iteration (3.2) converges to the solution of $S x=f$.

The aim of this paper is to prove the equivalence between the convergence of above two iterations when $T$ is generalized Lipschitz and $\Phi$-strongly pseudocontractive mapping in real uniformly smooth Banach spaces. Our results improve and extend the corresponding known results. For this purpose, we need the following Lemmas.

Lemma $1.1([4])$. Let $E$ be a real Banach space, then for all $x, y \in E$, there exists $j(x+y) \in J(x+y)$ such that

$$
\|x+y\|^{2} \leq\|x\|^{2}+2\langle y, j(x+y)\rangle .
$$

Lemma $1.2([8])$. Let $\left\{\rho_{n}\right\}_{n=0}^{\infty}$ be a nonnegative sequence which satisfies the following inequality

$$
\rho_{n+1} \leq\left(1-\lambda_{n}\right) \rho_{n}+\sigma_{n}, n \geq 0,
$$

where $\lambda_{n} \in(0,1), \sum_{n=0}^{\infty} \lambda_{n}=\infty$ and $\sigma_{n}=o\left(\lambda_{n}\right)$. Then $\rho_{n} \rightarrow 0$ as $n \rightarrow \infty$.

\section{Main results}

Theorem 2.1. Let $E$ be a real uniformly smooth Banach space and $D$ be a nonempty closed convex subset of $E$, let $T: D \rightarrow D$ be a generalized Lipschitz and $\Phi$-strongly pseudocontractive map. Let $q$ be a fixed point of $T$ in D. Suppose that $\left\{u_{n}\right\}_{n=0}^{\infty}$ and $\left\{x_{n}\right\}_{n=0}^{\infty}$ are defined by (1.5) and (1.6) respectively, with the iterative parameters $\left\{a_{n}\right\}_{n=0}^{\infty}$ and $\left\{b_{n}\right\}_{n=0}^{\infty}$ satisfying: $a_{n}, b_{n} \rightarrow 0$ as $n \rightarrow \infty$; $\sum_{n=0}^{\infty} a_{n}=\infty$. Then the following two assertions are equivalent:

(i) Mann iteration (1.5) converges to the fixed point of T;

(ii) Ishikawa iteration (1.6) converges to the fixed point of $T$.

Proof. By the definition of the strongly pseudocontractive map, we know that the fixed point of $T$ is unique. If the Ishikawa iteration (1.6) converges to the fixed point $q \in F(T)$, then set $b_{n}=0$, we can get the convergence of the Mann iteration (1.5). Conversely, we only want to prove (i) $\Rightarrow$ (ii), i.e., $\left\|u_{n}-q\right\| \rightarrow 0$ as $n \rightarrow \infty \Rightarrow\left\|x_{n}-q\right\| \rightarrow 0$ as $n \rightarrow \infty$. Without loss of generality, we assume that $\left\|u_{n}-q\right\| \leq 1$ for all $n \geq 0$.

Applying Lemma 1.1, (1.5), (1.6), and (1.3), we have

$$
\begin{aligned}
& \left\|x_{n+1}-u_{n+1}\right\|^{2} \\
\leq & \left(1-a_{n}\right)^{2}\left\|x_{n}-u_{n}\right\|^{2}+2 a_{n}\left\langle T y_{n}-T u_{n}, j\left(x_{n+1}-u_{n+1}\right)\right\rangle \\
\leq & \left(1-a_{n}\right)^{2}\left\|x_{n}-u_{n}\right\|^{2}+2 a_{n}\left\langle T y_{n}-T u_{n}, j\left(y_{n}-u_{n}\right)\right\rangle \\
& +2 a_{n}\left\langle T y_{n}-T u_{n}, j\left(x_{n+1}-u_{n+1}\right)-j\left(y_{n}-u_{n}\right)\right\rangle \\
\leq & \left(1-a_{n}\right)^{2}\left\|x_{n}-u_{n}\right\|^{2}+2 a_{n}\left(\left\|y_{n}-u_{n}\right\|^{2}-\Phi\left(\left\|y_{n}-u_{n}\right\|\right)\left\|y_{n}-u_{n}\right\|\right)
\end{aligned}
$$




$$
\begin{aligned}
& +2 a_{n}\left\|T y_{n}-T u_{n}\right\| \cdot\left\|j\left(\frac{x_{n+1}-u_{n+1}}{1+\left\|x_{n}-u_{n}\right\|}\right)-j\left(\frac{y_{n}-u_{n}}{1+\left\|x_{n}-u_{n}\right\|}\right)\right\| \\
& \cdot\left(1+\left\|x_{n}-u_{n}\right\|\right) \\
\leq & \left(1-a_{n}\right)^{2}\left\|x_{n}-u_{n}\right\|^{2}+2 a_{n}\left(\left\|y_{n}-u_{n}\right\|^{2}-\Phi\left(\left\|y_{n}-u_{n}\right\|\right)\left\|y_{n}-u_{n}\right\|\right) \\
& +2 a_{n} L\left(1+\left\|y_{n}-u_{n}\right\|\right) A_{n}\left(1+\left\|x_{n}-u_{n}\right\|\right),
\end{aligned}
$$

where $A_{n}=\left\|j\left(\frac{x_{n+1}-u_{n+1}}{1+\left\|x_{n}-u_{n}\right\|}\right)-j\left(\frac{y_{n}-u_{n}}{1+\left\|x_{n}-u_{n}\right\|}\right)\right\| \rightarrow 0$ as $n \rightarrow \infty$. Indeed, since

$$
\begin{aligned}
& \left\|\frac{x_{n+1}-u_{n+1}}{1+\left\|x_{n}-u_{n}\right\|}\right\| \\
\leq & \frac{\left(1-a_{n}\right)\left\|x_{n}-u_{n}\right\|+a_{n}\left\|T y_{n}-T u_{n}\right\|}{1+\left\|x_{n}-u_{n}\right\|} \\
\leq & 1-a_{n}+\frac{a_{n} L\left(1+\left\|y_{n}-u_{n}\right\|\right)}{1+\left\|x_{n}-u_{n}\right\|} \\
\leq & 1-a_{n}+\frac{a_{n} L\left(1+\left(1-b_{n}\right)\left\|x_{n}-u_{n}\right\|+b_{n}\left\|T x_{n}-u_{n}\right\|\right)}{1+\left\|x_{n}-u_{n}\right\|} \\
\leq & 1-a_{n}+\frac{a_{n} L\left(1+\left(1-b_{n}\right)\left\|x_{n}-u_{n}\right\|+b_{n}\left(\left\|T x_{n}-T u_{n}\right\|+\left\|T u_{n}-u_{n}\right\|\right)\right)}{1+\left\|x_{n}-u_{n}\right\|} \\
\leq & 1-a_{n}+\frac{a_{n} L\left(1+\left(1-b_{n}\right)\left\|x_{n}-u_{n}\right\|+b_{n}\left(L\left(1+\left\|x_{n}-u_{n}\right\|\right)+\left\|T u_{n}-T q\right\|+\left\|u_{n}-q\right\|\right)\right)}{1+\left\|x_{n}-u_{n}\right\|} \\
\leq & 1-a_{n}+a_{n} L\left(2-b_{n}+2 b_{n} L+b_{n}(1+L)\left\|u_{n}-q\right\|\right),
\end{aligned}
$$

and

$$
\begin{aligned}
& \left\|\frac{y_{n}-u_{n}}{1+\left\|x_{n}-u_{n}\right\|}\right\| \\
\leq & \frac{\left(1-b_{n}\right)\left\|x_{n}-u_{n}\right\|+b_{n}\left\|T x_{n}-u_{n}\right\|}{1+\left\|x_{n}-u_{n}\right\|} \\
\leq & \frac{\left(1-b_{n}\right)\left\|x_{n}-u_{n}\right\|+b_{n}\left(\left\|T x_{n}-T u_{n}\right\|+\left\|u_{n}-T u_{n}\right\|\right)}{1+\left\|x_{n}-u_{n}\right\|} \\
\leq & \frac{\left(1-b_{n}\right)\left\|x_{n}-u_{n}\right\|+b_{n}\left(L\left(1+\left\|x_{n}-u_{n}\right\|\right)+\left\|u_{n}-q\right\|+\left\|T u_{n}-T q\right\|\right)}{1+\left\|x_{n}-u_{n}\right\|} \\
\leq & \frac{\left(1-b_{n}\right)\left\|x_{n}-u_{n}\right\|+b_{n}\left(L\left(1+\left\|x_{n}-u_{n}\right\|\right)+\left\|u_{n}-q\right\|+L\left(1+\left\|u_{n}-q\right\|\right)\right)}{1+\left\|x_{n}-u_{n}\right\|} \\
\leq & 1-b_{n}+b_{n} L+b_{n}\left(L+(1+L)\left\|u_{n}-q\right\|\right),
\end{aligned}
$$

so sequences $\left\{\frac{x_{n+1}-u_{n+1}}{1+\left\|x_{n}-u_{n}\right\|}\right\}$ and $\left\{\frac{y_{n}-u_{n}}{1+\left\|x_{n}-u_{n}\right\|}\right\}$ are bounded, and

$$
\begin{aligned}
& \left\|\frac{\left(x_{n+1}-u_{n+1}\right)-\left(y_{n}-u_{n}\right)}{1+\left\|x_{n}-u_{n}\right\|}\right\| \\
\leq & \left|b_{n}-a_{n}\right|+2 a_{n} L+2 a_{n} b_{n} L^{2}+a_{n} b_{n} L\left\|u_{n}-q\right\| \\
& +a_{n} L+b_{n}+b_{n} L+b_{n}(1+L)\left\|u_{n}-q\right\| \rightarrow 0
\end{aligned}
$$

as $n \rightarrow \infty$. Using uniformly continuity of $j$ on bounded subset of $E$, then $A_{n} \rightarrow 0$ as $n \rightarrow \infty$.

Again using Lemma 1.1, (1.6), and (1.3), we obtain

$$
\begin{aligned}
& \left\|y_{n}-u_{n}\right\|^{2} \\
\leq & \left(1-b_{n}\right)^{2}\left\|x_{n}-u_{n}\right\|^{2}+2 b_{n}\left\langle T x_{n}-T u_{n}, j\left(y_{n}-u_{n}\right)\right\rangle \\
& +2 b_{n}\left\langle T u_{n}-u_{n}, j\left(y_{n}-u_{n}\right)\right\rangle \\
\leq & \left(1-b_{n}\right)^{2}\left\|x_{n}-u_{n}\right\|^{2}+2 b_{n}\left\langle T x_{n}-T u_{n}, j\left(x_{n}-u_{n}\right)\right\rangle \\
& +2 b_{n}\left\langle T x_{n}-T u_{n}, j\left(y_{n}-u_{n}\right)-j\left(x_{n}-u_{n}\right)\right\rangle+2 b_{n}\left\|T u_{n}-u_{n}\right\| \cdot\left\|y_{n}-u_{n}\right\|
\end{aligned}
$$




$$
\begin{aligned}
\leq & \left(1-b_{n}\right)^{2}\left\|x_{n}-u_{n}\right\|^{2}+2 b_{n}\left(\left\|x_{n}-u_{n}\right\|^{2}-\Phi\left(\left\|x_{n}-u_{n}\right\|\right)\left\|x_{n}-u_{n}\right\|\right) \\
& +2 b_{n}\left\|T x_{n}-T u_{n}\right\| \cdot\left\|j\left(y_{n}-u_{n}\right)-j\left(x_{n}-u_{n}\right)\right\| \\
& +2 b_{n}\left\|T u_{n}-u_{n}\right\| \cdot\left\|y_{n}-u_{n}\right\| \\
\leq & \left(1+b_{n}^{2}\right)\left\|x_{n}-u_{n}\right\|^{2}-2 b_{n} \Phi\left(\left\|x_{n}-u_{n}\right\|\right)\left\|x_{n}-u_{n}\right\| \\
& +2 b_{n} L\left(1+\left\|x_{n}-u_{n}\right\|\right) \cdot\left\|j\left(\frac{y_{n}-u_{n}}{1+\left\|x_{n}-u_{n}\right\|}\right)-j\left(\frac{x_{n}-u_{n}}{1+\left\|x_{n}-u_{n}\right\|}\right)\right\| \\
& \cdot\left(1+\left\|x_{n}-u_{n}\right\|\right)+2 b_{n}\left\|T u_{n}-u_{n}\right\| \cdot\left\|y_{n}-u_{n}\right\| \\
\leq & \left(1+b_{n}^{2}\right)\left\|x_{n}-u_{n}\right\|^{2}-2 b_{n} \Phi\left(\left\|x_{n}-u_{n}\right\|\right)\left\|x_{n}-u_{n}\right\| \\
& +2 b_{n} L\left(1+\left\|x_{n}-u_{n}\right\|\right)^{2} B_{n}+2 b_{n}\left\|T u_{n}-u_{n}\right\| \cdot\left\|y_{n}-u_{n}\right\| \\
\leq & \left(1+b_{n}^{2}\right)\left\|x_{n}-u_{n}\right\|^{2}-2 b_{n} \Phi\left(\left\|x_{n}-u_{n}\right\|\right)\left\|x_{n}-u_{n}\right\| \\
& +4 b_{n} L\left(1+\left\|x_{n}-u_{n}\right\|^{2}\right) B_{n}+2 b_{n}\left\|T u_{n}-u_{n}\right\| \cdot\left\|y_{n}-u_{n}\right\| \\
\leq & \left(1+b_{n}^{2}+4 b_{n} B_{n} L\right)\left\|x_{n}-u_{n}\right\|^{2}-2 b_{n} \Phi\left(\left\|x_{n}-u_{n}\right\|\right)\left\|x_{n}-u_{n}\right\|+4 b_{n} B_{n} L \\
& +2 b_{n}\left\|T u_{n}-u_{n}\right\| \cdot\left\|y_{n}-u_{n}\right\|,
\end{aligned}
$$

where $B_{n}=\left\|j\left(\frac{y_{n}-u_{n}}{1+\left\|x_{n}-u_{n}\right\|}\right)-j\left(\frac{x_{n}-u_{n}}{1+\left\|x_{n}-u_{n}\right\|}\right)\right\| \rightarrow 0$ as $n \rightarrow \infty$ (By the uniformly continuous of $j$ on a bounded set). Furthermore, we have the following estimates as a part of (2.5)

$$
\begin{aligned}
& \left\|y_{n}-u_{n}\right\| \\
= & \left\|\left(1-b_{n}\right)\left(x_{n}-u_{n}\right)+b_{n}\left(T x_{n}-u_{n}\right)\right\| \\
= & \left\|\left(1-b_{n}\right)\left(x_{n}-u_{n}\right)+b_{n}\left(T x_{n}-T u_{n}\right)+b_{n}\left(T u_{n}-u_{n}\right)\right\| \\
\leq & \left(1-b_{n}\right)\left\|x_{n}-u_{n}\right\|+b_{n} L\left(1+\left\|x_{n}-u_{n}\right\|\right)+b_{n}\left\|T u_{n}-T q\right\|+b_{n}\left\|u_{n}-q\right\| \\
\leq & \left(1-b_{n}\right)\left\|x_{n}-u_{n}\right\|+b_{n} L\left(1+\left\|x_{n}-u_{n}\right\|\right)+b_{n} L\left(1+\left\|u_{n}-q\right\|\right)+b_{n}\left\|u_{n}-q\right\| \\
\leq & 2 b_{n} L+\left(1-b_{n}+b_{n} L\right)\left\|x_{n}-u_{n}\right\|+\left(b_{n}+b_{n} L\right)\left\|u_{n}-q\right\|,
\end{aligned}
$$

and

$$
\begin{aligned}
& 2 b_{n}\left\|T u_{n}-u_{n}\right\| \cdot\left\|y_{n}-u_{n}\right\| \\
\leq & 2 b_{n}\left(\left\|T u_{n}-T q\right\|+\left\|u_{n}-q\right\|\right) \\
& \cdot\left(2 b_{n} L+\left(1-b_{n}+b_{n} L\right)\left\|x_{n}-u_{n}\right\|+\left(b_{n}+b_{n} L\right)\left\|u_{n}-q\right\|\right) \\
\leq & 2 b_{n}\left(L+(1+L)\left\|u_{n}-q\right\|\right) \\
& \cdot\left(2 b_{n} L+\left(1-b_{n}+b_{n} L\right)\left\|x_{n}-u_{n}\right\|+\left(b_{n}+b_{n} L\right)\left\|u_{n}-q\right\|\right) \\
= & 2 b_{n} C_{n}\left(2 b_{n} L+D_{n}\left\|x_{n}-u_{n}\right\|+E_{n}\right) \\
\leq & 2 b_{n} C_{n}\left(2 b_{n} L+E_{n}\right)+b_{n} C_{n} D_{n}\left(1+\left\|x_{n}-u_{n}\right\|^{2}\right),
\end{aligned}
$$

where $C_{n}=L+(1+L)\left\|u_{n}-q\right\|, D_{n}=1-b_{n}+b_{n} L, E_{n}=\left(b_{n}+b_{n} L\right)\left\|u_{n}-q\right\|$.

Substituting (2.7) into (2.5), we obtain that

$$
\left\|y_{n}-u_{n}\right\|^{2} \leq\left(1+b_{n}^{2}+4 b_{n} B_{n} L+b_{n} C_{n} D_{n}\right)\left\|x_{n}-u_{n}\right\|^{2}
$$




$$
\begin{aligned}
& +b_{n} C_{n}\left(4 b_{n} L+2 E_{n}+D_{n}\right)+4 b_{n} B_{n} L \\
\leq & \left(1+W_{n}\right)\left\|x_{n}-u_{n}\right\|^{2}+V_{n},
\end{aligned}
$$

where $W_{n}=b_{n}^{2}+4 b_{n} B_{n} L+b_{n} C_{n} D_{n}, V_{n}=b_{n} C_{n}\left(4 b_{n} L+2 E_{n}+D_{n}\right)+4 b_{n} B_{n} L$. We now estimate $2 a_{n} L\left(1+\left\|y_{n}-u_{n}\right\|\right) A_{n}\left(1+\left\|x_{n}-u_{n}\right\|\right)$ in formula (2.1). Using (2.6), we have

$$
\text { (2.9) } \begin{aligned}
& 2 a_{n} L\left(1+\left\|y_{n}-u_{n}\right\|\right) A_{n}\left(1+\left\|x_{n}-u_{n}\right\|\right) \\
\leq & 2 a_{n} A_{n} L\left(1+2 b_{n} L+\left(1-b_{n}+b_{n} L\right)\left\|x_{n}-u_{n}\right\|+\left(b_{n}+b_{n} L\right)\left\|u_{n}-q\right\|\right) \\
& \cdot\left(1+\left\|x_{n}-u_{n}\right\|\right) \\
\leq & 2 a_{n} A_{n} L\left(1+2 b_{n} L+E_{n}+D_{n}\left\|x_{n}-u_{n}\right\|\right) \cdot\left(1+\left\|x_{n}-u_{n}\right\|\right) \\
\leq & 2 a_{n} A_{n} L\left(1+2 b_{n} L+E_{n}+\left(1+2 b_{n} L+E_{n}+D_{n}\right)\left\|x_{n}-u_{n}\right\|\right. \\
& \left.\quad+D_{n}\left\|x_{n}-u_{n}\right\|^{2}\right) \\
\leq & 2 a_{n} A_{n} L\left(1+2 b_{n} L+E_{n}+2\left(1+2 b_{n} L+E_{n}+D_{n}\right)\left\|x_{n}-u_{n}\right\|\right. \\
\quad & \left.\quad D_{n}\left\|x_{n}-u_{n}\right\|^{2}\right) \\
\leq & 2 a_{n} A_{n} L\left(1+2 b_{n} L+E_{n}+\left(1+2 b_{n} L+E_{n}+D_{n}\right)\left(1+\left\|x_{n}-u_{n}\right\|^{2}\right)\right. \\
& \left.\quad+D_{n}\left\|x_{n}-u_{n}\right\|^{2}\right) \\
\leq & 2 a_{n} A_{n} L\left(2+4 b_{n} L+2 E_{n}+D_{n}+\left(1+2 b_{n} L+E_{n}+2 D_{n}\right)\left\|x_{n}-u_{n}\right\|^{2}\right) \\
\leq & 2 a_{n} A_{n} F_{n} L\left(1+\left\|x_{n}-u_{n}\right\|^{2}\right),
\end{aligned}
$$

where $F_{n}=2+4 b_{n} L+2 E_{n}+2 D_{n}$.

Taking (2.9) into (2.1), we get that

$$
\begin{aligned}
& \left\|x_{n+1}-u_{n+1}\right\|^{2} \\
\leq & \left(1-a_{n}\right)^{2}\left\|x_{n}-u_{n}\right\|^{2}+2 a_{n}\left(\left\|y_{n}-u_{n}\right\|^{2}-\Phi\left(\left\|y_{n}-u_{n}\right\|\right)\left\|y_{n}-u_{n}\right\|\right) \\
& +2 a_{n} A_{n} F_{n} L\left(1+\left\|x_{n}-u_{n}\right\|^{2}\right) .
\end{aligned}
$$

Set $\eta=\inf _{n \geq 0} \frac{\Phi\left(\left\|y_{n}-u_{n}\right\|\right)}{1+\left\|y_{n}-u_{n}\right\|}$. Then $\eta=0$. If such is not the case, we assume that $\eta>0$, and choose a $\delta>0$ such that $0<\delta<\min \{1, \eta\}$. Then we have $\Phi\left(\left\|y_{n}-u_{n}\right\|\right) \geq \delta+\delta\left\|y_{n}-u_{n}\right\| \geq \delta\left\|y_{n}-u_{n}\right\|$.

It follows from (2.10) and (2.8) that

$$
\begin{aligned}
& \left\|x_{n+1}-u_{n+1}\right\|^{2} \\
\leq & \left(1-a_{n}\right)^{2}\left\|x_{n}-u_{n}\right\|^{2}+2 a_{n}(1-\delta)\left(\left(1+W_{n}\right)\left\|x_{n}-u_{n}\right\|^{2}+V_{n}\right) \\
& +2 a_{n} A_{n} F_{n} L\left(1+\left\|x_{n}-u_{n}\right\|^{2}\right) . \\
\leq & \left(1-a_{n}\left(2 \delta-a_{n}-2 W_{n}-2 A_{n} F_{n} L\right)\right)\left\|x_{n}-u_{n}\right\|^{2}+2 a_{n}(1-\delta) V_{n}+2 a_{n} A_{n} F_{n} L .
\end{aligned}
$$


Since $a_{n}+2 W_{n}+2 A_{n} F_{n} L \rightarrow 0$ as $n \rightarrow \infty$, then there exists $n_{0}$ such that, for all $n>n_{0}$, we have $a_{n}+2 W_{n}+2 A_{n} F_{n} L<\delta$, this leads to

$$
\begin{aligned}
& \left\|x_{n+1}-u_{n+1}\right\|^{2} \\
\leq & \left(1-a_{n} \delta\right)\left\|x_{n}-u_{n}\right\|^{2}+2 a_{n}(1-\delta) V_{n}+2 a_{n} A_{n} F_{n} L
\end{aligned}
$$

for all $n>n_{0}$. By Lemma 1.2, we get $\left\|x_{n}-u_{n}\right\| \rightarrow 0$ as $n \rightarrow \infty$. Again formula (2.6), we have $\left\|y_{n}-u_{n}\right\| \rightarrow 0$ as $n \rightarrow \infty$, contradicting condition $\eta>0$. Hence $\eta=0$. Since $\Phi$ is strictly increasing and continuous with $\Phi(0)=0$, then there exists a subsequence $\left\{y_{n_{i}}-u_{n_{i}}\right\}$ of $\left\{y_{n}-u_{n}\right\}$ such that $\lim _{i \rightarrow \infty}\left\|y_{n_{i}}-u_{n_{i}}\right\|=0$. On using (1.6), we obtain that

$$
\begin{aligned}
& \left\|y_{n}-u_{n}\right\| \\
= & \left\|\left(1-b_{n}\right)\left(x_{n}-u_{n}\right)+b_{n}\left(T x_{n}-u_{n}\right)\right\| \\
\geq & \left(1-b_{n}\right)\left\|x_{n}-u_{n}\right\|-b_{n} L\left(1+\left\|x_{n}-u_{n}\right\|\right)-b_{n}\left\|T u_{n}-T q\right\|-b_{n}\left\|u_{n}-q\right\| \\
\geq & \left(1-b_{n}\right)\left\|x_{n}-u_{n}\right\|-b_{n} L\left(1+\left\|x_{n}-u_{n}\right\|\right)-b_{n} L\left(1+\left\|u_{n}-q\right\|\right)-b_{n}\left\|u_{n}-q\right\| \\
\geq & -2 b_{n} L+\left(1-b_{n}-b_{n} L\right)\left\|x_{n}-u_{n}\right\|-\left(b_{n}+b_{n} L\right)\left\|u_{n}-q\right\| .
\end{aligned}
$$

Here, without loss of generality, we assume that $1-b_{n}-b_{n} L>0$ for all $n \geq 0$. Thus (2.13) implies that

$$
\begin{aligned}
& \left\|y_{n_{i}}-u_{n_{i}}\right\|+2 b_{n_{i}} L+\left(b_{n_{i}}+b_{n_{i}} L\right)\left\|u_{n_{i}}-q\right\| \\
\geq & \left(1-b_{n_{i}}-b_{n_{i}} L\right)\left\|x_{n_{i}}-u_{n_{i}}\right\| \geq 0 .
\end{aligned}
$$

Since $\lim _{i \rightarrow \infty}\left\|y_{n_{i}}-u_{n_{i}}\right\|=0, \lim _{n \rightarrow \infty} b_{n}=0, \lim _{n \rightarrow \infty}\left\|u_{n}-q\right\|=0$, then we have $\lim _{i \rightarrow \infty}\left\|x_{n_{i}}-u_{n_{i}}\right\|=0$. Let $0<\epsilon<1$ be given, choose $N$ such that, for all $i \geq N,\left\|x_{n_{i}}-u_{n_{i}}\right\|<\epsilon,\left\|y_{n_{i}}-u_{n_{i}}\right\|<\epsilon, a_{n_{i}}<\frac{\epsilon}{2 \epsilon+2 L(1+\epsilon)}$, $b_{n_{i}}<\frac{\epsilon}{6 \epsilon+8 L \epsilon+8 L}$ and $a_{n_{i}}+4 A_{n_{i}} F_{n_{i}} L<\Phi\left(\frac{\epsilon}{4}\right)\left(\frac{\epsilon}{4}\right),\left\|u_{n_{i}}-q\right\|<\epsilon$. In this case, we can prove that $\left\|x_{n_{i}+m}-u_{n_{i}+m}\right\|<\epsilon . \forall m=1,2,3, \ldots$. First we prove that $\left\|x_{n_{i}+1}-u_{n_{i}+1}\right\|<\epsilon$. Suppose this is not the case. Then $\left\|x_{n_{i}+1}-u_{n_{i}+1}\right\| \geq \epsilon$. From (1.6), we obtain that

$$
\begin{aligned}
& \left\|x_{n_{i}}-u_{n_{i}}\right\| \\
\geq & \left\|x_{n_{i}+1}-u_{n_{i}+1}\right\|-a_{n_{i}}\left\|x_{n_{i}}-u_{n_{i}}\right\|-a_{n_{i}}\left\|T y_{n_{i}}-T u_{n_{i}}\right\| \\
\geq & \epsilon-a_{n_{i}} \epsilon-a_{n_{i}} L\left(1+\left\|y_{n_{i}}-u_{n_{i}}\right\|\right) \\
\geq & \epsilon-a_{n_{i}} \epsilon-a_{n_{i}} L(1+\epsilon) \\
= & \epsilon-a_{n_{i}}(\epsilon+L(1+\epsilon)) \\
\geq & \frac{\epsilon}{2}
\end{aligned}
$$

and

$$
\begin{aligned}
& \left\|y_{n_{i}}-u_{n_{i}}\right\| \\
\geq & \left(1-b_{n_{i}}\right)\left\|x_{n_{i}}-u_{n_{i}}\right\|-b_{n_{i}}\left\|T x_{n_{i}}-u_{n_{i}}\right\| \\
\geq & \left(1-b_{n_{i}}\right)\left\|x_{n_{i}}-u_{n_{i}}\right\|-b_{n_{i}}\left\|T x_{n_{i}}-T u_{n_{i}}\right\|-b_{n_{i}}\left\|T u_{n_{i}}-u_{n_{i}}\right\|
\end{aligned}
$$




$$
\begin{aligned}
\geq & \left(1-b_{n_{i}}\right)\left\|x_{n_{i}}-u_{n_{i}}\right\|-b_{n_{i}} L\left(1+\left\|x_{n_{i}}-u_{n_{i}}\right\|\right) \\
& -b_{n_{i}}\left(\left\|T u_{n_{i}}-T q\right\|+\left\|u_{n_{i}}-q\right\|\right) \\
\geq & \left(1-b_{n_{i}}\right)\left\|x_{n_{i}}-u_{n_{i}}\right\|-b_{n_{i}} L\left(1+\left\|x_{n_{i}}-u_{n_{i}}\right\|\right) \\
& \left.-b_{n_{i}}(L+(1+L))\left\|u_{n_{i}}-q\right\|\right) \\
\geq & \left(1-b_{n_{i}}\right) \frac{\epsilon}{2}-b_{n_{i}} L(1+\epsilon)-b_{n_{i}}(L+(1+L) \epsilon) \\
= & \left(1-b_{n_{i}}\right) \frac{\epsilon}{2}-b_{n_{i}} L(1+\epsilon)-b_{n_{i}}(L+(1+L) \epsilon)>\frac{\epsilon}{4}
\end{aligned}
$$

for all $i \geq N$. From (2.10), we have

$$
\begin{aligned}
& \left\|x_{n_{i}+1}-u_{n_{i}+1}\right\|^{2} \\
\leq & \left(1-a_{n_{i}}\right)^{2}\left\|x_{n_{i}}-u_{n_{i}}\right\|^{2}+2 a_{n_{i}}\left(\left\|y_{n_{i}}-u_{n_{i}}\right\|^{2}-\Phi\left(\left\|y_{n_{i}}-u_{n_{i}}\right\|\right)\left\|y_{n_{i}}-u_{n_{i}}\right\|\right) \\
& +2 a_{n_{i}} A_{n_{i}} F_{n_{i}} L\left(1+\left\|x_{n_{i}}-u_{n_{i}}\right\|^{2}\right) \\
< & \left(1-a_{n_{i}}\right)^{2} \epsilon^{2}+2 a_{n_{i}} \epsilon^{2}-2 a_{n_{i}} \Phi\left(\frac{\epsilon}{4}\right) \frac{\epsilon}{4}+2 a_{n_{i}} A_{n_{i}} F_{n_{i}} L\left(1+\epsilon^{2}\right) \\
\leq & \epsilon^{2}+a_{n_{i}}\left(a_{n_{i}} \epsilon^{2}+4 A_{n_{i}} F_{n_{i}} L-2 \Phi\left(\frac{\epsilon}{4}\right) \frac{\epsilon}{4}\right) \\
\leq & \epsilon^{2}-a_{n_{i}} \Phi\left(\frac{\epsilon}{4}\right) \frac{\epsilon}{4} \\
< & \epsilon^{2},
\end{aligned}
$$

which is a contradiction. Hence $\left\|x_{n_{i}+1}-u_{n_{i}+1}\right\|<\epsilon$. Now we assume that $\left\|x_{n_{i}+m}-u_{n_{i}+m}\right\|<\epsilon$ holds. It follows from the above argument that $\| x_{n_{i}+m+1}$ $-u_{n_{i}+m+1} \|<\epsilon$. Therefore, this shows that $\lim _{n \rightarrow \infty}\left\|x_{n}-u_{n}\right\|=0$. Since $\lim _{n \rightarrow \infty}\left\|u_{n}-q\right\|=0$, and the inequality

$$
0 \leq\left\|x_{n}-q\right\| \leq\left\|x_{n}-u_{n}\right\|+\left\|u_{n}-q\right\| \rightarrow 0
$$

as $n \rightarrow \infty$. That is, $\lim _{n \rightarrow \infty}\left\|x_{n}-q\right\|=0$.

Remark 1. In [2], Bogin showed that $T$ is strongly pseudocontractive if and only if $(I-T)$ is strongly accretive, where $I$ denotes the identity operator. By this fact, then $T$ is $\Phi$-strongly pseudocontractive if and only if $(I-T)$ is $\Phi$-strongly accretive. Thus we have the following results.

Theorem 2.2. Let $E$ be a real uniformly smooth Banach space. Assume that $T: E \rightarrow E$ is a generalized Lipschitz and $\Phi$-strongly accretive operator. For any given $f \in E$, define $S: E \rightarrow E$ by $S x=f-T x+x$ for all $x \in E$. Suppose that $q$ is the solution of equation $T x=f$. Let $\left\{a_{n}\right\}_{n=0}^{\infty}$ and $\left\{b_{n}\right\}_{n=0}^{\infty}$ be two real sequences $[0,1]$ in satisfying the conditions: (i) $a_{n}, b_{n} \rightarrow 0$ as $n \rightarrow \infty$; (ii) $\sum_{n=0}^{\infty} a_{n}=\infty$. Define the Ishikawa iterative sequence $\left\{x_{n}\right\}_{n=0}^{\infty}$ generated from an arbitrary $x_{0} \in E$ by

$$
\left\{\begin{array}{c}
x_{n+1}=\left(1-a_{n}\right) x_{n}+a_{n} S y_{n}, n \geq 0, \\
y_{n}=\left(1-b_{n}\right) x_{n}+b_{n} S x_{n}, n \geq 0,
\end{array}\right.
$$


and Mann iteration sequence $\left\{u_{n}\right\}_{n=0}^{\infty}$ generated from an arbitrary $u_{0} \in E$ by

$$
u_{n+1}=\left(1-a_{n}\right) u_{n}+a_{n} S u_{n}, n \geq 0 .
$$

Then the following two assertions are equivalent:

(i) Mann iteration (2.19) converges to the solution of equation $T x=f$;

(ii) Ishikawa iteration (2.18) converges to the solution of equation $T x=f$. $\mathrm{Or}$

(i) Mann iteration (2.19) converges to the fixed point of $S$;

(ii) Ishikawa iteration (2.18) converges to the fixed point of $S$.

Proof. Since $T$ is generalized Lipschitzian and $\Phi$-strongly accretive operator, by the definition of $S$, then we know that $S$ is generalized Lipschitzian $\Phi$-strongly pseudocontractive mapping. Applying Theorem 2.1, we obtain conclusion of Theorem 2.2.

Remark 2. Theorem 2.1 and Theorem 2.2 extend and improve Theorem 2.1 and Corollary 3.1 of [3] in the following sense:

(i). The hypotheses that both "the bounded subset $D$ in [3, Theorem 2.1]" and " $\left\{x_{n}\right\}$ is bounded in [3, Corollary 3.1]" are replaced by the more general condition generalized Lipschitz maps.

(ii). The strongly pseudocontractive in [3, Theorem 2.1] and the strongly accretive operators [3, Corollary 3.1$]$ are replaced by $\Phi$-strongly pseudocontractive and $\Phi$-strongly accretive operators respectively.

Remark 3. A map $T$ is said to be uniformly $\psi$-pseudocontractive if there exists a function $\psi: \Re^{+} \rightarrow \Re^{+}$which is strictly continuous increasing with $\psi(0)=0$, and a $j(x-y) \in J(x-y)$ such that

$$
\langle T x-T y, j(x-y)\rangle \leq\|x-y\|^{2}-\psi(\|x-y\|)
$$

for all $x, y \in D(T)$. By the remark 1 , then $T$ is uniformly $\psi$-accretive if and only if $I-T$ is uniformly $\psi$-pseudocontractive. Hence using the same methods, we may also obtain that Theorem 2.1 and Theorem 2.2 hold for the more general class of the generalized Lipschitzian uniformly $\psi$-pseudocontractive and uniformly $\psi$-accretive operators individually.

Acknowledgments. The author is very grateful to the referees for their valuable comments and good suggestions.

\section{References}

[1] V. Barbu, Nonlinear Semigroups and Differential Equations in Banach Spaces, Editura Academiei Republicii Socialiste Romania, Bucharest; Noordhoff International Publishing, Leiden, 1976

[2] J. Bogin, On Strict Pseudo-Contractions and a Fixed Point Theorem, Technion Preprint MT-29, Haifa, 1974.

[3] F. E. Browder, Nonlinear operators and nonlinear equations of evolution in Banach spaces, Nonlinear functional analysis (Proc. Sympos. Pure Math., Vol. XVIII, Part 2, Chicago, Ill., 1968), pp. 1-308. Amer. Math. Soc., Providence, R. I., 1976.

[4] K. Deimling, Nonlinear Functional Analysis, Springer-Verlag, Berlin, 1985. 
[5] T. Kato, Nonlinear semigroups and evolution equations, J. Math. Soc. Japan 19 (1967), $508-520$.

[6] B. E. Rhoades and S. M. Soltuz, The equivalence of Mann iteration and Ishikawa iteration for non-Lipschitzian operators, Int. J. Math. Math. Sci. (2003), no. 42, 2645-2651.

[7] _ On the equivalence of Mann and Ishikawa iteration methods, Int. J. Math. Math. Sci. (2003), no. 7, 451-459.

[8] X. Weng, Fixed point iteration for local strictly pseudo-contractive mapping, Proc. Amer. Math. Soc. 113 (1991), no. 3, 727-731.

Department of Mathematics and Physics

ShiJiazhuang Railway Institute

ShiJiazhuang 050043, P. R. China

E-mail address: xuezhiqun@126.com 